\title{
A TOOL TO MONITOR THE NETWORK QUALITY OF SERVICE
}

\author{
Fabien Michaut \\ and Francis Lepage \\ Centre de Recherche en Automatique de Nancy (CRAN) \\ CNRS UMR 7039, Université Henri Poincaré, Nancy 1, BP 239, \\ F-54506 Vandoeuvre-lès-Nancy Cedex \\ fabien.michaut@cran.uhp-nancy.fr
}

\begin{abstract}
This paper is introducing a monitoring tool of networks Quality of Service (QoS). The aim of the tool is to carry out active measurements on IP networks. Its design is adapted to a QoS architecture that was presented in a previous work (Michaut and Lepage, 2002), but it can also be used separately for network management. The Unified Modeling Language is used to model it.
\end{abstract}

Keywords: Network monitoring, network metrology, Quality of Service, QoS engineering.

\section{Introduction}

In a previous work (Michaut and Lepage, 2002), we have introduced a Quality of Service (QoS) architecture based on the Prayer architecture (Bharghavan and Gupta, 1997). It allows applications to adapt their execution to their operating environment. The adaptation to resources fluctuations requires the measurement of the QoS.

This paper presents a tool to monitor networks QoS. It is designed to be integrated in the QoS architecture, but it can be used separately for network management purpose for example ${ }^{1}$.

The paper is organized as follows: Section one reminds the constraints the monitoring tool has to comply with due to its integration in the QoS architecture. The design of the monitoring tool is then introduced in section two. Section three describes the monitoring agent and the coordinator entities. Finally, we present our conclusions and explain our future work.

The original version of this chapter was revised: The copyright line was incorrect. This has been corrected. The Erratum to this chapter is available at DOI: 10.1007/978-0-387-35620-4_43 


\section{Integration constraints}

This part lists the constraints the tool has to supply with due to its integration in the QoS architecture. For further explanations on these constraints, please see (Michaut and Lepage, 2002).

Measurements can be carried out actively and/or passively. Active measurements are usually performed while sending probe packets to the receiver through the network. Passive measurements are made in observing the useful traffic. Due to the adaptation framework, active measurements have to be compulsorily done.

An other important point in monitoring is the frequency of the measurement process. This frequency will be determined according to the intrinsic frequency of the application and has then to be configurable.

The architecture needs to know path characteristics in the two ways. Thus the monitoring component has to be able to perform measurements in both directions of an end-to-end communication.

Additionally, variations in resources have to be detected by the endsystem that initiates the QoS adaptation. It means that a monitoring component needs to request measurements of other monitoring components of other end-systems.

The monitoring tool that we propose in this paper is dealing with twoway active network measurements. Measured parameters are the oneway delay, the round-trip time, the jitter, the loss rate and the available bandwidth. The tool is planned to work on IP networks using TCP or UDP transport protocols. Frequency of the measurement process is configurable. Its design allows two tools (of two different end-systems) to cooperate and network passive measurement and end-system resources measurement procedures to be added in the future.

\section{Monitoring tool design}

\subsection{Overview}

When an end-system needs measurements to be made between itself and another one, a measurement procedure has to be set up by the two concerned monitoring tools. The monitoring tool is designed according to a client-server architecture: The system requesting the measurement procedure is called the client, the system answering the request is called the server.

The proposed monitoring tool is only focusing on active measurements at that time. This technique requires a probes sender and a probes receiver. By definition, the probes sender is called the client sensor, the 
probes receiver is called the server sensor. The setup of a measurement procedure corresponds then to the setup of a sensors couple.

Figure 1 illustrates the case in which the end-system $A$ has requested the setup of a sensors couple of the end-system $B$ ( $A$ is the client, B is the server). In the first case (figure 1a), the delay from $A$ to $B$ has to be measured. The client sensor (probes sender) is located on $A$ and the server sensor (probes receiver) on $B$. In the second case (figure $1 b$ ), the delay from $\mathrm{B}$ to $\mathrm{A}$ has to be measured. Then, the client sensor is located on $B$ and the server sensor on A. Note that the client/server concepts for sensors and end-systems are totally independent. Furthermore, the same procedure is used to measure the one-way delay in both directions. Changing the direction of the measurement procedure is possible by inversing the sensors place. This remark is valid for every one-way parameters (jitter, loss, etc.). In addition, it is to be noted that an end-system can be client and server simultaneously. This would be the case in the previous example if an end-system $\mathrm{C}$ had requested a sensors setup of A.

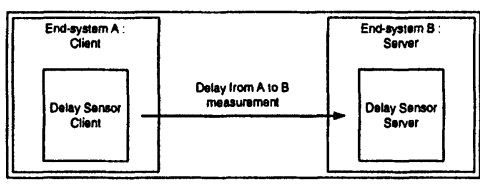

(a)

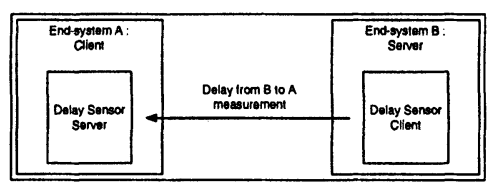

(b)

Figure 1. Client and server end-systems, client and server sensors

\subsection{Modeling}

The monitoring tool is modeled in an object-oriented approach using the Unified Modeling Language (UML). The UML classes relative to the sensors are shown on the figure 2: the monitoring tool implements sensors to measure delay, jitter and loss (classes DelayJitterLossClient and DelayJitterLossServer), round-trip time (classes RttClient and RttServer) and available bandwidth(classes AvailableBwClient and AvailableBwServer). For example, the abstract class Sensor defines the sensor identifier, the transport protocol which the probes have to use, etc. These parameters are common to all sensors. The class PassiveSensor allows passive measurement sensors to be added in the future. The classes ActiveAvailableBw, ActiveDelayJitterLoss and ActiveRtt include all the attributes and operations which are common to the corresponding sensors couples. 
For all sensors, probes sending and receiving process are started by calling clients and servers operations start. The operations sendMeasurements are used by sensors to send measurements back to their monitoring agent (see 3 ). The operations evaluateMonitoring Traffic calculate the throughput of the traffic that is generated to perform measurements.

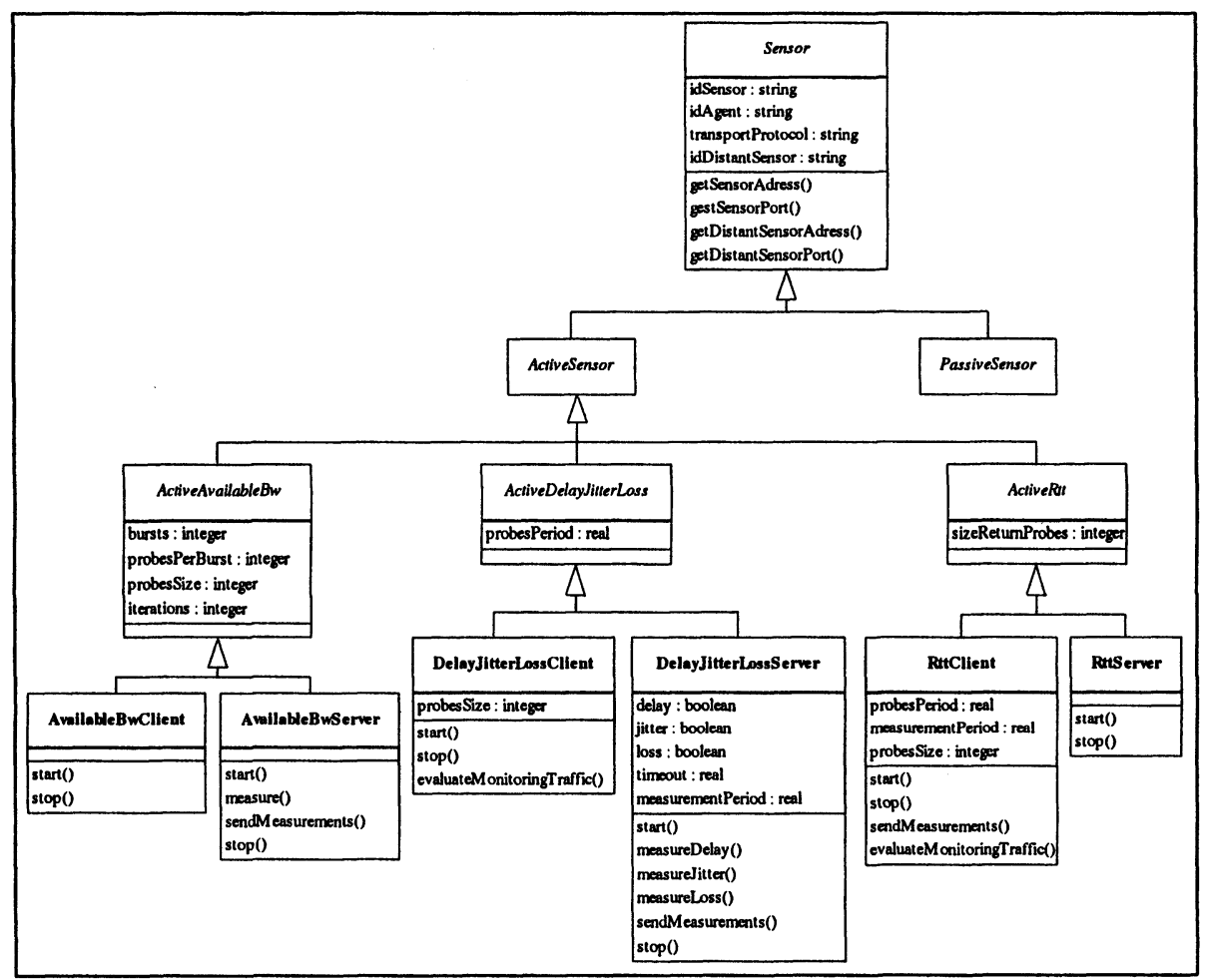

Figure 2. Sensors UML Class Diagram

As an example, the delay, jitter and loss sensors are described in the following : The so-called 'one-way delay' is the time a packet takes to go from the source to the destination. To measure it, a probe packet is stamped with the current time and sent to the destination. At the destination, the probe timestamp and the destination clock are read out. The delay is then equal to the difference between the two values.Jitter is the variation of the delay and is usually computed as the delay difference between two consecutive probe packets ${ }^{2}$. The loss rate is equal to the number of non-received packets divided by the total number of sent packets. Metrics of these parameters are proposed respectively in (Almes et al., 1999a), (Demichelis and Chimento, 2000) and (Almes et al., 1999b). 
These three parameters are measured by setting up a client sensor which sends periodically probes to a server sensor according to the attribute probesPeriod. The probes size is indicated by the attribute probesSize. Probes contain their departure timestamp and a sequence number that allow the server sensor to determine the delay and the loss ${ }^{3}$. The booleans attributes delay, jitter and loss inform the server of the parameter(s) it has to measure.Measurements are calculated periodically by the server sensor according to the attribute measurementPeriod.

\section{Monitoring agent and coordinator concepts}

Several applications can run on an end-system. Each of them can request different measurements of the monitoring tool between the endsystem and other end-systems. Entities are then required to organize sensors functioning (ie. to initiate sensors set up, to specify attributes values, to present measurements to the architecture, etc.)

These needed entities are the monitoring agents. Each application is combined with one monitoring agent. A monitoring agent can manage several sensors couples.

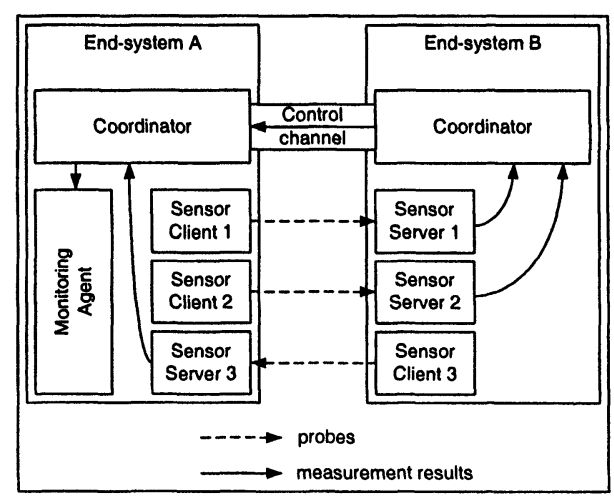

Figure 3. Measurements flows

An other entity called the coordinator is present on each end-system. It enables the sensors and the agents that are on its end-system to work with the sensors and the agents that are on an other end-system. The coordinator has to set up or remove sensors couples at the request of a monitoring agent, in collaboration with the coordinator of the target end-system.

The communication between two coordinators is performed through a channel called the control channel. Messages are for example 'START SENSOR', 'STOP SENSOR', 'SENSOR CREATED', etc. When a sen- 
sor has to send its measurements to its agent for example (see figure 3), it sends the message to its coordinator. The coordinator determines the location of the target agent and forwards the message accordingly ${ }^{4}$. The procedure is similar when an agent has to send a message to a sensor.

\section{Conclusions and future work}

The purpose of this paper was to present a network monitoring tool. This tool is modeled to allow its integration in our QoS architecture, but it can be used in a separate way to carry out measurements on networks. Measurements are performed actively.The tool is designed according to a client/server architecture. We used UML to model it and we are currently developing it. For execution performances and to exploit the interest of object-oriented modeling techniques, we use $\mathrm{C} / \mathrm{C}++$ programming language. Next we will have to evaluate the performances of the tool and refine the measurement techniques consequently.

\section{Acknowledgments}

This work is funded by the Lorraine region.

\section{Notes}

1. Network monitoring is more generally a very actual research theme (Owezarski, 2001).

2. Jitter can also be defined as the difference between the maximum and minimum delay or the average deviation of the delay.

3. Knowing the sending period and the sequence numbers of previous probes, the server sensor can determine the highest sequence number he should have received at time $t$.

4. If the target agent is on the local system, the coordinator forwards the message to it directly. If the target agent is on a remote system, the coordinator sends the message to the coordinator of the concerned system.

\section{References}

Almes, G., Kalidindi, S., and Zekauskas, M. (1999a). A one-way delay metric for IPPM, RFC 2679.

Almes, G., Kalidindi, S., and Zekauskas, M. (1999b). A one-way packet loss metric for IPPM, RFC 2680.

Bharghavan, V. and Gupta, V. (1997). A framework for application adaptation in mobile computing environments. In IEEE Compsac'97.

Demichelis, C. and Chimento, P. (2000). Instantaneous packet delay variation metric for IPPM, internet draft.

Michaut, F. and Lepage, F. (2002). A QoS architecture for application execution adaptation. In 3rd ACIS International Conference on Software Engineering, Artificial Intelligence, Networking and Parallel/Distributed Computing (SNPD'02).

Owezarski, P. (2001). Que nous dit la métrologie sur le futur d'Internet? In JRES 2001, Lyon. 